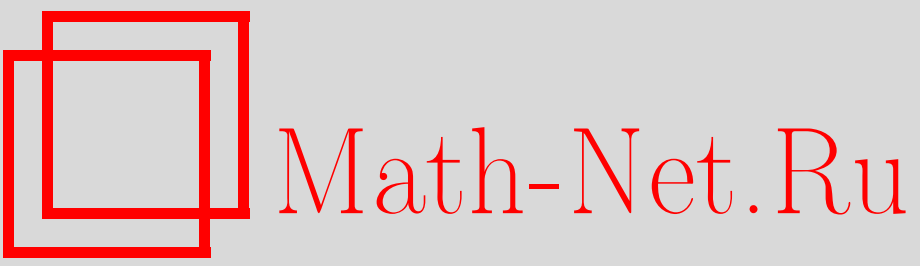

S. N. Mayburov, Photonic communications in biological systems, Вестн. Сам. гос. техн. ун-та. Сер. Физ.-мат. науки, 2011, выпуск 2(), 260-265

DOI: https://doi.org/10.14498/vsgtu952

Использование Общероссийского математического портала Math-Net.Ru подразумевает, что вы прочитали и согласны с пользовательским соглашением

http://www. mathnet.ru/rus/agreement

Параметры загрузки:

IP: 107.22 .136 .117

26 апреля 2023 г., 12:35:04 
UDC 517.958:57; MSC: 92C05; 92C37, 62P10

\section{PHOTONIC COMMUNICATIONS IN BIOLOGICAL SYSTEMS}

\section{S. N. Mayburov}

P. N. Lebedev Physical Institute, Russian Academy of Sciences, 53, Leninskiy Prospect, Moscow, 117924, Russia.

E-mail: mayburov@sci.lebedev.ru

The mechanism of communications between distant bio-systems by means of optical and UV photons is studied. In the proposed model the bio-systems perform such communications radiating the photons in form of short periodic bursts, which were observed experimentally for fish and frog eggs. In this case the communication algorithm can be similar to the exchange of binary encoded data in the computer nets via the noisy channels. The analysis of experimental data on the radiation of fish eggs confirm our hypothesis and reveals the main features of signal encoding.

Key words: biological signaling, photon radiation, signal encoding.

Introduction. Currently, the term 'biophotons' is attributed to the optical and UV photons emitted by the living bio-systems in the processes which are different from standard chemi-luminescence. Their systematic measurements by the low-noise electronic photo-detectors was started about 1978 [1,2]; the biophoton production (BP) in optical and close UV range was established now for large amount of bio-systems $[3,4]$. It was found that its rate and other parameters are quite sensitive to the characteristics of bio-system and its development. Because of it, the biophoton measurements are succesfully applied now in many different fields from medical diagnostics to agriculture and ecology [2].

The energy spectrum of biophotons is nearly constant within optical and close UV range practically for all studied bio-systems, so it essentially differs from the spectra expected for the system with the temperature about $300 \mathrm{~K}$, which in this range should fall on 15 orders of magnitude $[2,3]$. The detailed BP mechanism is still unknown, but such molecular excitations can be stipulated by the biochemical reactions in which oxygen atoms are bound to the proteins and acids.

The typical bio-photon rates are quite low, however, the multiple experiments evidence that such radiation can perform the effective signaling between distant bio-systems. In particular, being radiated by the developing bio-system and absorbed by the similar one at the distance about several $\mathrm{cm}$, it can rise the rate of cell mitosis in it up to $30 \%$ relative to the standard values. This phenomenon called mitogenetic effect (ME) is extensively studied in the last years $[2,3]$. Note that the artificial constant illumination by the visible light, even $10^{4}$ times more intense, can't induce the comparable gain. The communications of some other types were reported also; for the bio-systems in the state of abrupt stress or slow destruction (apoptosis) such radiation can change the state of other bio-systems in the similar depressive way $[2,5]$.

Until now, most of BP properties, as well as their communication mechanism, can't be described within the standard framework of cellular biology. In our previous paper the simple model of information exchange between the biosystems by means of noncoherent photons was proposed [6]. We've assumed that the main features of such communications are similar to the information exchange

Sergey N. Mayburov, Senior Scientific Scholar, Lab. of Elementary Particles. 
between the distant computers by the binary encoded messages. This hypothesis is prompted by the experimental results which show that the biophoton radiation consists of narrow quasi-periodic bursts (figs. 1,2), so its time spectra is similar to the sequence of electronic or photonic pulses which transfer information in the computer communication channels $[1,4]$. To check this hypothesis, we performed the analysis of data for the radiation from fish eggs, it permits to study the algorithms, by which such bio-system encode the produced photon signals.

Communication Model. Before considering the photon exchange between the distant bio-systems, it's worth to discuss how such communications can be per-

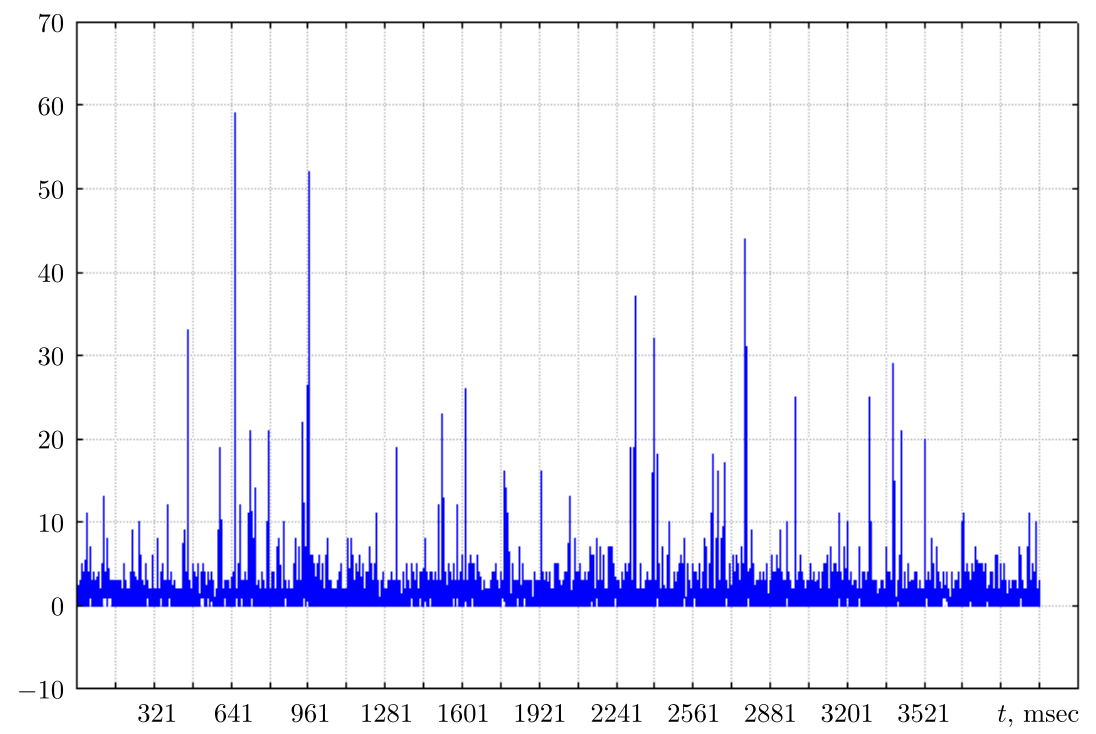

Fig. 1. Example of biophoton time spectra for 16 development stage, full time scale $400 \mathrm{sec}$

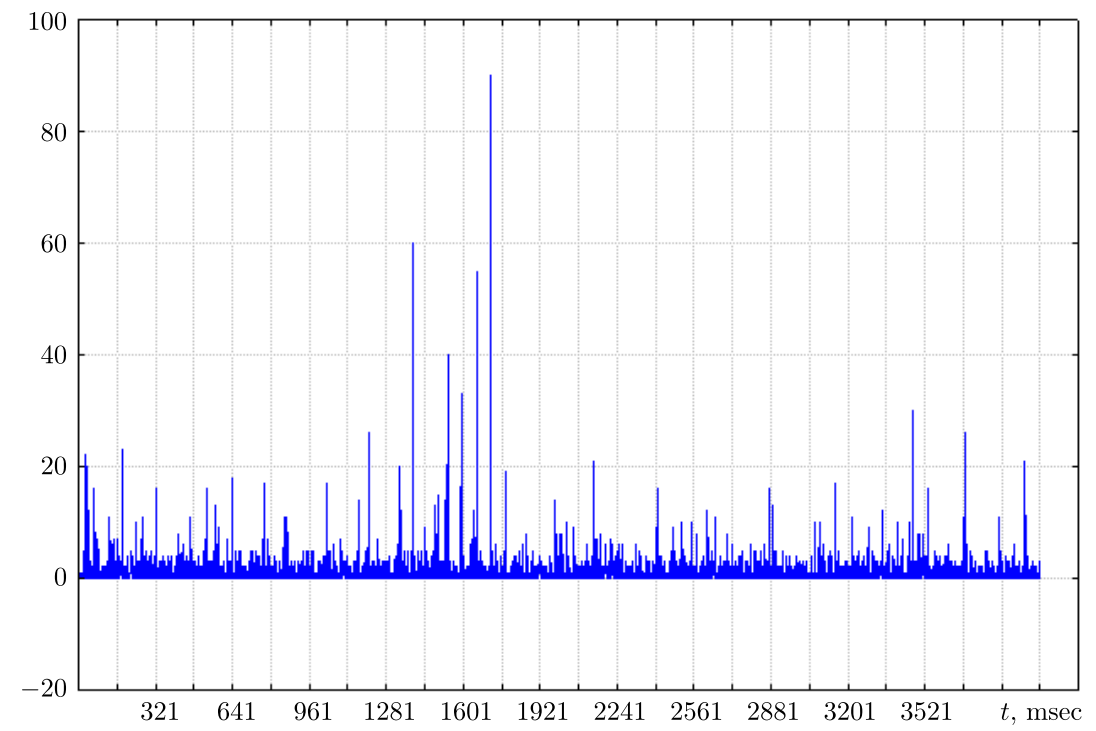

Fig. 2. Example of biophoton time spectra for 30 development stage, full time scale 400 sec 
formed between the parts of the same dense bio-system. The optical and UV excitations in the dense media exist as the quasi-particles called excitons which can spread freely through the whole media volume [7]. They are strongly coupled with e-m field, so they can be produced during the photons absorption by the media with high efficiency, the inverse process results in the photons emission from the system volume. It's established experimentally now that the excitons play the important role in the energy transfer inside the bio-systems, in particular, during the photosynthesis in plants and bacteria [8]. BP mechanism related to the nonlinear excitons spreading along the protein molecules was studied in [9]. In our model the excitations of biological media as the whole play the main role in biophotons generation and absorption. We don't consider here any model Hamiltonian, which is planned for the future, however, for such distances it's inevitably should have the solitonic properties [7]. The recent experiments show the permanent existence of optical excitations in living bio-systems, their rate is many orders larger than expected for the thermal equilibrium at $300 \mathrm{~K}$. Hence it can't be excluded that the energy losses of exciton due to its rescattering by the media can be compensated from the interactions with such excitations which transfer their energy to the exciton. Thereon, we suppose that the excitons spread freely over all the volume of bio-system.

As was noticed above, BP rate is quite low, about 10 photons $/ \mathrm{cm}^{2} \mathrm{sec}$ from the surface of large, dense bio-system. If such e-m field isn't coherent, then it described as the stochastic ensemble of photons. Then at its best the absorption of single photon or narrow bunch of photons can be detected by the bio-system as the single independent 'click' or one bit of information, analogously to standard photodetector devices. This is the photocounting regime of e-m field detection well-known in quantum optics [10]. We suppose that the same approach is applicable also for the excitons produced and absorbed in the same bio-system. Under this assumptions, the exciton signaling between two parts of the same bio-system and photon signalling between two distant bio-systems can be quite similar, so the mechanism proposed below for exciton signalling is applicable also to photonic one with minor changes

In our approach we suppose that the signals which controls the cell mitosis and other functions can be similar to the standard discrete (binary) encoded messages transferred between two computers via the noisy communication channels [6]. The nature of such similarity can be understood from the simple common sense reasoning without exploit of information theory machinery. Plainly, for the low exciton or photon radiation rates, typical for bio-systems, the most important problem for the effective signal transfer is to suppress the background. Even for the bio-systems in complete darkness it induced by many sources, like the natural radioactivity, luminescent chemical impurities, etc. Consequently, as the main criteria characterizing the efficiency of information exchange, the signal to noise ratio $K_{O}$ can be used, i.e. the ratio of registrated 'clicks' induced by the bio-system signals and the background. It's natural to suppose that the evolution of living species made the information exchange by means of excitons or photon radiation/absorption practically optimal. The average rate of background radiation normally should be constant in time, so for given bio-system with the limited radiation intensity the optimal method to achieve high $K_{O}$ level is to make the main bulk of the bio-system radiation to be concentrated inside the short time intervals, i.e. the bio-system radiation should be in the form of bursts which would encode the signals transferred to other bio-systems. The experiments with fish 
eggs, fibroblast cells and other bio-systems demonstrated that the biophotons are radiated by the short-time (less than $1 \mathrm{msec}$ ) quasi-periodic bursts $[1,4]$; the typical time spectrum for fish eggs is shown on figs. 1, 2 .

The influence of biophoton exchange on the organism development was found for many species, in particular their detailed study was performed for the eggs of loach fish (Misgurnus fossilis). Note that for the egg colony produced by the fish at once it's favorable for the larvae survival, if all eggs would develop with the same speed. However, the small variations of temperature and water flow over colony volume and other external factors tend to violate this condition. It seems that the biophoton signaling between distant eggs of the same colony restore their simultaneous development. The results for the optical contacts during 30-50 min. between the samples of fish eggs A, B of slightly different age demonstrate the significant synchronization of their development ([1] and refs. therein). However, it was found also that the optical contacts between the fish eggs of significantly different ages result in the serious violations of development in both samples, for fish eggs at early stages the development can simply stop. Those results evidence that the photon signals emitted by fish eggs at different development stages can have the essentially different structure.

Comparison with Experimental Results. The possible signal structure was exploited for the experimental data on the optical radiation of loach fish eggs. The studied sample is the colony about 200 eggs, which is confined in the quartz container filled with water, their radiation from the container surface was measured by the photo-multiplier. Its intensity was summed over the nonoverlapping consequent time intervals (bins) with the duration $0.1 \mathrm{sec}$; the experimental run normally consists of $6 \cdot 10^{3}$ such bins [1]. The measurements were performed for the different development stages, from the earliest ones (cleavage) to the latest 33-35 stages, preceding the free larvae appearance; the average stage duration is about 1.5 hour. The background was measured for the container filled with water only.

Since the background is supposedly stochastic and there is no time correlations between its intensity at different time moments, then the periodicity or some other time correlations between such bursts would help to discriminate the background more effectively. Simultaneously, the variations of such correlations can also encode the different signals send by the bio-system. It was supposed that such encoding is performed by the methods and algorithms similar to the standard ones used for noisy communication channels [6]. In this framework, as the model example it can be supposed that the separate message consists of $N$ bursts of the same height $I_{r}$ with time interval $T_{r}$ between them, such messages are divided by the periods of 'silence' $T_{s}$ when the detectable radiation is similar to the stochastic background, i.e. each message constitutes the burst cluster and such messages divided by $T_{S}$ are repeated many times [6]. Plainly, for the realistic bio-systems those parameters would have the significant stochastic spread $\sigma_{I}$, $\sigma_{T}, \ldots$ around the average values.

First, we studied the discrimination of fish eggs radiation against the background, for which the burst distribution should be stochastic with the expectation values of burst amplitude $\bar{I}$ and time interval $\bar{T}$. The fish eggs radiation is expected to have more periodical structure, for example, if the burst radiation of fish eggs is strictly periodical, i.e. it can be approximated as:

$$
A(t)=I_{r} \delta\left(t-n T_{r}\right)
$$


for arbitrary integer $n$, then its fourier time spectra is equal to:

$$
a_{\nu}=\int_{0}^{\infty} A(\tau) \cos \nu \tau d \tau=I_{r} \delta\left(\nu-\pi l / T_{r}\right)
$$

for integer $l$ and $0<l \leqslant \infty$. Thus, the burst periodicity is reflected in fourier spectra, whereas for stochastic background $\bar{a}_{\nu}=\bar{I}$, i.e. is constant function with some fluctuations around its average value, so the difference of those two cases is essential. Hence Fourier analysis of time spectra can be exploited to select the periodic radiation from the background; STATISTICA 7.0 packet was used for the data processing.

The preliminary analysis has shown that the typical duration of biophoton message is about $10^{2}$ sec and $\bar{N}=8.5$, so the density of fourier time spectra $f(\nu)$ is calculated for each consequent set of 1200 bins in given run separately, i.e. 5 spectra for each run. In this template the density $f(\nu)=a_{\nu}^{2}+b_{\nu}^{2}$ where $b_{\nu}$ is the value of corresponding fourier integral (1) for $\sin \nu \tau$. Then for each spectra $f$ its autocorrelation:

$$
g(\mu)=\int_{0}^{\infty} f(\nu) f(\nu+\mu) d \nu / \int_{0}^{\infty} f^{2}(\nu) d \nu
$$

was calculated. The value of integral $R$ of $g$ module over such set is used as the selection criteria:

$$
R=\int|g(\mu)| d \mu .
$$

It was found that for arbitrary $R$ threshold which selects about $80 \%$ of fish eggs runs, only $16 \pm 4 \%$ backgorund runs are selected for $12-16$ stages and $23 \pm 6 \%$ for 30-34 stages. It evidences that the fish eggs radiation has more periodical structure than the stochastic background, the possible candidate for such periodical signal (message) is shown on fig. 2. If to characterize the radiation structure in terms of our model example then the individual messages have the length about $0.6 \div 1.5 \cdot 10^{2}$ sec and they are interspersed by the periods of silence about $4 \div 7 \cdot 10^{2}$ sec. Each message consists of $6-14$ distinct high bursts, despite significant fluctuations, their amplitudes $I$ demonstrate gaussian-like dependence on the time distance from the message centre (see fig. 2). In each individual message the time intervals $T$ between the bursts with $I$ higher than some threshold $I_{0}$ are nearly the same with some dispersion or differ by the whole number, i.e. are equal to $2 T$, $3 T$, etc. Yet $T$ average value can differ from one message to another significantly, about factor 1.8 on the average.

Concerning the signal distinction between the different development stages, the analysis of data indicates that the most pronounced change suffers $T$ average value. $T$ distributions for the bursts with $I$ higher than 20 units are shown for 1216 and 30-34 stages on fig. 3. Meanwhile, the average number of bursts is nearly the same for both cases, whereas the average burst amplitude $\bar{I}$ for $30-34$ stages is about 15-20\% larger than for 12-16 stages, yet the overlap of their $I$ distributions is essentially larger than for $T$ distributions. The obtained distinctions explain, probably, the difference in the influence of radiation from fish eggs of different age on the 'detector' samples of fish eggs. Despite that the difference of signal parameters is only statistical, the multiple repetition of such messages can be eventually percepted by the 'detector' fish eggs as the different instructions which 


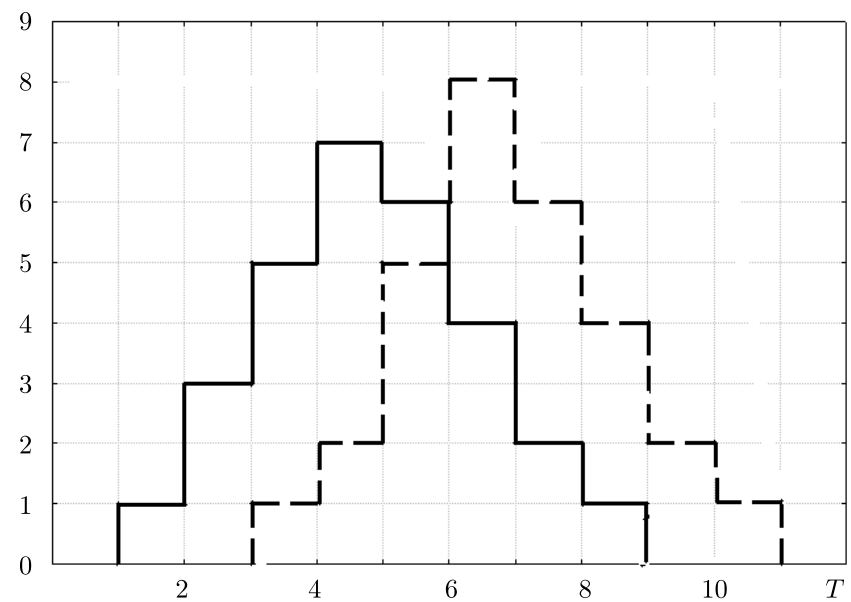

Fig. 3. $T$ distribution for fish eggs radiation: broken line $-12-16$ stages; solid line $-30-35$ stages

would be fulfilled during their subsequent development. It seems that the most important for their encoding is $T$ difference, because the burst amplitude produce mainly the threshold effect, i.e. only the condition like $I>I_{0}$ is accounted. If those conclusions will be confirmed by further experiments, it would mean that the main encoding algorithm for fish eggs radiation has the analogue realization, despite the produced signal is constituted by the sequence of discrete bursts.

\section{REFERENCES}

1. Beloussov L. $V$ Exploring the dynamic background of the developmental processes and cell reactions with the use of an ultraweak photon emission // Biosystems, 2003. Vol. 68, no. 2-3. Pp. 199-212.

2. VanWijk R. Bio-photons and Bio-communication // J. Sci. Explor., 2001. Vol. 15, no. 2. Pp. 183-209.

3. Popp F.A., Ruth B., Bahr W., Bohm J., Grass P., Grolig G., Rattemeyer M., Schmidt H. G., Wulle P. Emission of visible and ultraviolet radiation by active biological systems // Collect. Phenomena, 1981. Vol. 3. Pp. 187-214.

4. Beloussov L. V., Burlakov A.B., Louchinskaia N.N. Biophotonic patterns of optical interactions between fish eggs and embryos // Indian J. Exp. Biol., 2003. Vol.41, no. 5. Pp. 424-430.

5. Farhadi A., Shaikha M., Engena Ph., Fieldsa J.Z., Keshavarziana A. Evidence for nonchemical, non-electrical intercellular signaling in intestinal epithelial cells // Bioelectrochemistry, 2008. Vol. 71, no. 2. Pp. 142-148.

6. Mayburov S. Biophoton production and communications / In: Proc. of Int. Conf. on Nanotechnology and Nanomaterials. Moscow: MGOU Publishing, 2009. Pp. 351-358.

7. Davidov A. Solitons in molecular systems / Mathematics and its Applications. Dortreht: Kluwer, 1985. 419 pp.

8. Engel G.S., Calhoun T.R., Read E.L.; Ahn T.-K., Mančal T., Cheng Y.-C., Blankenship R. E., Fleming G. R. Evidence for wavelike energy transfer through quantum coherence in photosynthetic systems // Nature, 2007. Vol. 446, no. 7137. Pp. 782-786.

9. Brizhik L., Scordino A., Triglia A., Musumeci F. Delayed luminescence of biological systems arising from correlated many-soliton states // Phys. Rev. E, 2001. Vol. 64, no. 3, 031902. 9 pp.

10. Glauber R. J. Quantum Optics. New York: Academic Press, 1969. 759 pp.

Original article submitted 25/II/2011; revision submitted 15/III/2011. 